\title{
The effects of progressive sleep loss on a lexical decision task: Response lapses and response accuracy
}

\author{
HARVEY BABKOFF \\ Bar-Ilan University, Ramat-Gan, Israel
}

and

\author{
SANDER G. GENSER, HELEN C. SING, DAVID R. THORNE, and FREDERICK W. HEGGE \\ Walter Reed Army Institute of Research, Washington, DC
}

\begin{abstract}
A lexical decision task was used in a paradigm testing the effects of sleep loss and fatigue on performance during a 72-h period of sleep deprivation. The data were partitioned into categories of response lapses, response accuracy, and the signal detection measures of discriminability ( $\left.\mathrm{d}^{\prime}\right)$ and bias $(\beta)$. Response lapses increased as a function of sleep loss and were fitted best by a composite equation with a major linear component and a minor rhythmic component. Response accuracy decreased as a function of sleep loss, with the rate of decrease being greater for nonwords than for words. Although d' was higher for right visual field (RVF), it decreased for both fields almost linearly as a function of sleep deprivation. The rate of decrease for RVF stimulation was greater than for left visual field (LVF) stimulation. $\beta$ did not change monotonically as a function of sleep loss, but showed strong circadian rhythmicity, indicating that it was not differentially affected by sleep loss per se.
\end{abstract}

The psychological and physiological consequences of human fatigue and sleep loss have been studied for nearly a century (Patrick \& Gilbert, 1896). Although the experimental paradigm has changed little, research has emphasized different aspects of sleep and sleep loss (Johnson, 1982; Johnson \& Naitoh, 1974; Meddis, 1982). Meddis (1982) noted that there is no general theory to explain how sleep loss affects information-processing ability.

Several summary reviews indicate that performance decrement in cognitive tasks is most severe when tasks are prolonged or inherently boring. Studies have demonstrated that, by rewarding appropriate performance or maintaining high motivation, task performance can be sustained even after long hours of sleep deprivation (e.g., Wilkinson, 1964). Wide mood swings are observed and perceptual distortions and/or hallucinations are reported after extended sleep deprivation (Babkoff, Genser, Sing, Hegge, \& Thorne, 1985; Belenky, 1979; Johnson \& Naitoh, 1974; Meddis, 1982). These general conclusions aid in the design of studies, in task construction, and in the selection of variables to be manipulated. They are less helpful in the formulation of hypotheses concerning

The opinions or assertions contained herein are the private views of the authors and are not to be construed as official or as reflecting the views of the Department of the Army or the Department of Defense (para 4-3, AR 360-5). The authors' mailing addresses are: Bar Ilan University, 53100 Ramat Gan, Israel; and Department of Behavioral Biology, Walter Reed Army Institute of Research, Washington, DC 20307-5100. specific functional deficits resulting from fatigue and sleep loss.

Studying phenomena associated with the two cerebral hemispheres may aid in describing sleep-loss deficits. The lexical decision task is a useful mechanism for assessing linguistic hemispheric-functional specificity (Babkoff \& Ben-Uriah, 1983; Babkoff, Genser, \& Hegge, in press). We used the lexical decision task to test sleep-loss and fatigue effects on hemispheric-functional specificity and to provide a description of cognitive deficit related to known neuroanatomical substrates. If sleep provides a restorative rather than "scheduling" function for the brain (Horne, 1983), then any observed decline in discriminability associated with sleep loss should reflect decreased nervous system capacity rather than decreased motivation. If the functional deficit differs with respect to stimulation of the two visual hemifields, then the description of the effects of sleep loss may point to possible underlying mechanisms.

Recent studies of sleep-loss deficits have recognized that performance is not constant, but fluctuates rhythmically, requiring that studies of sleep loss be designed to assess these factors as well as those of fatigue (Monk et al., 1985; Thorne, Genser, Sing, \& Hegge, 1983). The present study provided for testing of lexical decision once every $2 \mathrm{~h}$, interspersed with other test packages over a 72-h period of sleep deprivation. Only the analyses of response omissions (lapses) and response accuracy are reported here. Response accuracy data are transformed into signal detection measures, $\mathrm{d}^{\prime}$ and $\beta$, and separately 
analyzed. Reaction time measures will be reported in a future publication.

\section{METHOD}

A description of the sleep-deprivation paradigm and the dynamic changes that occurred are presented in detail elsewhere (Babkoff, Thorne, Sing, Genser, Taube, \& Hegge, 1985). A brief overview of the experimental procedure is described here.

\section{Subjects}

Ten subjects participated in the sleep-deprivation experiment, which included testing on a variety of tasks. Two participated for $\mathbf{4 8} \mathrm{h}$ only, and equipment failures caused the loss of 10 out of 36 sessions for 2 other subjects. Full data sets of lexical decision for $72 \mathrm{~h}$ of sleep deprivation for 6 male subjects are presented in this report. Subjects, ranging in age from 18 to 24 years, were all high school graduates with $0-4$ years of college (median of 1 year), and had no history of diagnosable psychiatric disorder.

\section{Stimuli}

The stimulus pool consisted of 44 each of three-, four-, and five-letter English words and 44 each of three-, four-, and five-letter nonwords (constructed by randomly rearranging the letters of the words). The words were selected from Heise's (1978) compilation of 1,551 words from three separately published lists. The words selected were of high frequency according to the Thorndike Lorge criterion (i.e., frequency $\geq 50 / 1,000,000$ ).

\section{Apparatus}

Stimulus presentation and response recording were controlled via a Digital Equipment Corporation (DEC) PDP8/e minicomputer, and stimuli were presented on a DEC VR14 monitor. Stimuli were presented horizontally to the right or to the left visual fields (RVF, LVF). The visual angle of eccentricity measured from the position of the crossshaped fixation symbol at the center of the monitor to the nearest letter of the stimulus was $1^{\circ} 26^{\prime}$. Word and nonword stimuli were randomized by trial. Viewing was with the right eye only (left eye patched) at a distance of $1 \mathrm{~m}$ from the screen. The three-, four-, and five-letter stimuli subtended visual angles of approximately $1^{\circ} 9^{\prime}, 1^{\circ} 33^{\prime}$, and $2^{\circ} 4^{\prime}$, respectively. Stimulus duration was $150 \mathrm{msec}$, followed by a 100 -msec blanking flash to eliminate the slowly decaying image due to phosphor afterglow.

\section{Procedure}

Subjects were tested in pairs. Each spent approximately 3 days of the week prior to testing in training on the experimental tasks, and an additional day of the subsequent week in final preparation. The experiment always began on a Tuesday morning and continued until Friday morning. The subjects spent from 30 to $45 \mathrm{~min}$ of each hour in testing (duty cycle). This range reflects changes in the duty cycle which increased systematically over the course of the experiment (Babkoff, Thorne et al., 1985). Tests were conducted individually inside acoustically isolated room-sized chambers and were monitored by staff members via intercom and closed-circuit TV. At least two staff members were always in attendance.

A trial began with the appearance of a 1- to 2-sec fixation symbol in the center of the screen. The subject was instructed to focus on the symbol and not to move his eyes until after the response was made; eye position was monitored by closed-circuit TV. The stimulus was presented at the offset of the fixation symbol. The subject kept his right index finger on the middle of three electronic contact keys. If the finger was not placed on the center key or was removed from that key while the fixation symbol was still lighted, the stimulus was not presented. Subjects were instructed to respond "word" or "nonword" by moving the right index finger from the center key to the left or right key as quickly as possible.

The interval during which the subject could respond was $3 \mathrm{sec}$, timed from the onset of the stimulus. If a response was not made within $3 \mathrm{sec}$, a response lapse was recorded. The next trial began after a response, or at the termination of $5 \mathrm{sec}$ without a response.

\section{RESULTS}

All of the analyses were performed on three data sets: response lapses; response accuracy to word and nonword stimuli; and $\mathrm{d}^{\prime}$ and $\beta$ measures of the transformed response accuracy data using the correct responses to words as "hits" and the incorrect responses to nonwords as "false alarms."

\section{Response Lapses}

Any trial in which $3 \mathrm{sec}$ elapsed without a response was considered a response lapse. ${ }^{1}$ Response lapses were tabulated separately for trials in which words/nonwords were presented to each of the two hemifields, and were analyzed by a four-way ANOVA: type of stimulus (word/nonword) $\times$ visual hemifield $(\mathrm{RVF} / \mathrm{LVF}) \times$ number of days of sleep deprivation $x$ hour of day.

Results of the ANOVA indicated that all variables were statistically significant: type of stimulus $(F=16.13$, $\mathrm{p} \leq 0.01)$; visual hemifield $(\mathrm{F}=9.42, \mathrm{p} \leq 0.028)$; number of days of sleep deprivation $(F=13.89, p \leq 0.0013)$; hour of day $(F(8.59, p \leq 0.0001)$. No interactions were significant.

Response lapses increased by an average factor of 7.7 from Day 1 to Day 3 of sleep deprivation (from $2.6 \%$ to $20.6 \%$ ) and by a factor of 3.2 from $0800-1400$ to $2400-0600$ (from $6.54 \%$ to $20.7 \%$ ). The difference in response lapses between stimulation of RVF and LVF was $0.8 \%$ (11.8\% LVF, $11.0 \%$ RVF), and between words and nonwords was $1 \%$ (11.9\% nonwords, $10.9 \%$ words). Response lapses, averaged across the 6 subjects, are plotted in Figure 1 as a function of $72 \mathrm{~h}$ of sleep deprivation. Four curves are shown, representing data for each cell of the matrix: word/nonword and RVF/LVF stimulation. The four curves appear to have almost identical mono- 


\section{RESPONSE LAPSES AS A FUNCTION OF
SLEEP DEPRIVATION}

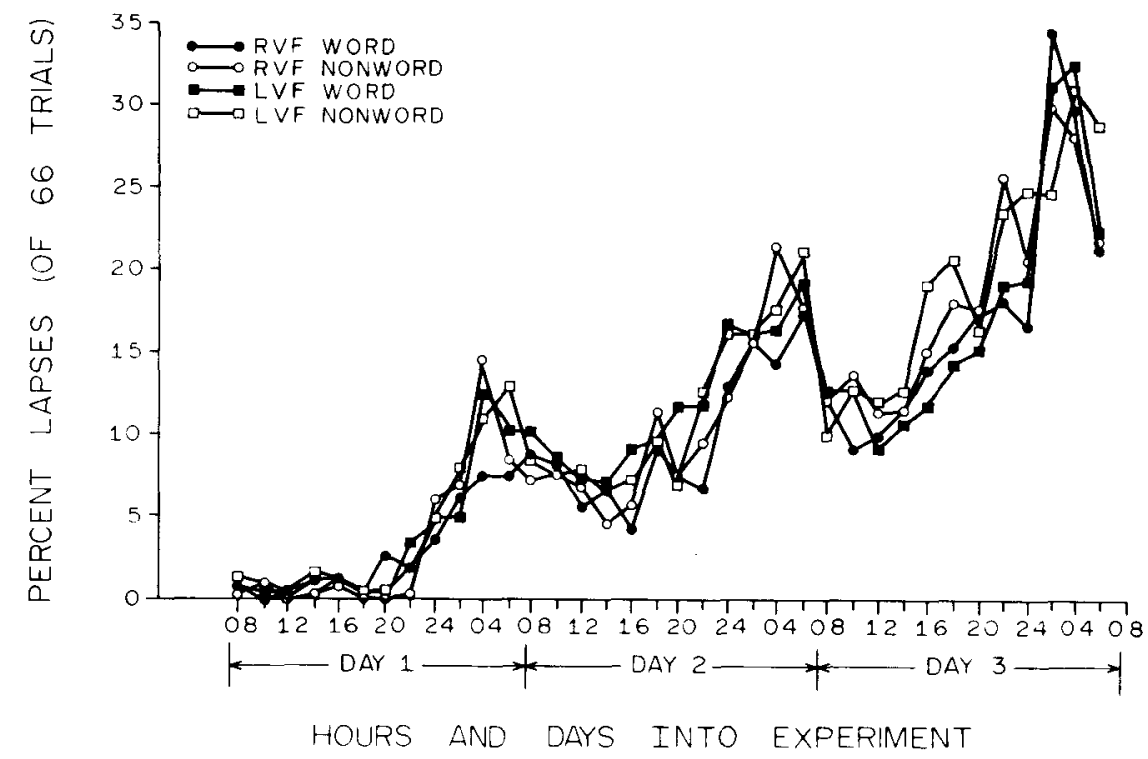

Figure 1. Relative frequency of response lapses as a function of hours of progressive sleep deprivation. Data are plotted for the four cells of the matrix: words and nonwords by right visual field (RVF) and left visual field (LVF).

tonic and rhythmic components that coincide during the last $12 \mathrm{~h}$ of sleep deprivation.

The curves were analyzed by multiple complex demodulation (MCD), (Redmond, Sing, \& Hegge, 1982; Sing, Genser, Babkoff, Thorne, \& Hegge, 1984; Sing, Redmond, \& Hegge, 1980; Sing, Thorne, Hegge, \& Babkoff, 1985). This technique allowed extraction of the overall trend, consisting of monotonic components and frequencies lower than the circadian; the circadian component; and higher frequency components. Regression analysis indicated that the overall trend accounted for an average of $83.8 \%$, the circadian component for an average of $11.4 \%$, and the two-cycles-per-day (cpd) component for approximately $2-3 \%$ of the variance in the raw data.

The MCD-derived trends then were fitted by linear and exponential equations. The best-fitting linear equation accounted for $98 \%$ of the variance in the trends themselves, and the best-fitting exponential equation accounted for $92 \%$. Since the trends account for $83.8 \%$ of the variance in the raw data, the linear and exponential equations account for approximately $82 \%$ and $77 \%$, respectively (Table 1A). Accordingly, the increase in response lapses, as a function of sleep deprivation, can best be described by a composite equation whose major component is monotonic and linear and whose minor component is circadian.

\section{Response Accuracy}

Response accuracy, defined as the percent of correct responses on trials in which a response was made, was tabulated separately for trials on which words/nonwords were presented to each of the two hemifields, and was analyzed by a four-way ANOVA [type of stimulus (word/nonword) $\times$ visual hemifield $(\mathrm{RVF} / \mathrm{LVF}) \times$ number of days of sleep deprivation $x$ hour of day]. All four main effects were significant. Response accuracy decreased from $82.7 \%$ to $64.6 \%$ over the 3 days of sleep

Table 1

Component Analysis (Complex Demodulation) of the Averaged Lexical Decision Data

\begin{tabular}{|c|c|c|c|c|}
\hline \multirow[b]{2}{*}{ Function } & \multicolumn{2}{|c|}{ Monotonic } & \multicolumn{2}{|c|}{ Rhythmic } \\
\hline & Linear & Exponential & Circadian & 2 Cycles/Day \\
\hline \multicolumn{5}{|c|}{ A. Response Lapses } \\
\hline Word/RVF & $80.0 \%$ & $76.4 \%$ & $10.6 \%$ & $3.8 \%$ \\
\hline Nonword/RVF & $82.6 \%$ & $77.0 \%$ & $11.3 \%$ & $2.1 \%$ \\
\hline Word/LVF & $80.2 \%$ & $73.4 \%$ & $13.1 \%$ & $2.5 \%$ \\
\hline Nonword/LVF & $84.8 \%$ & $80.1 \%$ & $10.7 \%$ & $1.0 \%$ \\
\hline \multicolumn{5}{|c|}{ B. Response Accuracy } \\
\hline Word/RVF & $39.0 \%$ & $37.4 \%$ & $35.3 \%$ & $9.0 \%$ \\
\hline Nonword/RVF & $88.7 \%$ & $85.4 \%$ & $3.4 \%$ & $1.7 \%$ \\
\hline Word/LVF & $28.0 \%$ & $29.0 \%$ & $16.3 \%$ & $9.0 \%$ \\
\hline Nonword/LVF & $87.2 \%$ & $85.0 \%$ & $4.6 \%$ & $0.3 \%$ \\
\hline \multicolumn{5}{|c|}{ C. Discriminability $\left(\mathrm{d}^{\prime}\right)$} \\
\hline RVF & $83.0 \%$ & $51.5 \%$ & $7.0 \%$ & $4.5 \%$ \\
\hline LVF & $88.9 \%$ & $40.2 \%$ & $4.1 \%$ & $1.0 \%$ \\
\hline \multicolumn{5}{|c|}{ D. Bias $(\beta)$} \\
\hline RVF & $2.2 \%$ & $2.5 \%$ & $42.6 \%$ & $13.1 \%$ \\
\hline LVF & $0.2 \%$ & $0.2 \%$ & $38.7 \%$ & $18.0 \%$ \\
\hline
\end{tabular}

Note-Amount of variance accounted for by monotonic and rhythmic components. 
deprivation ( $p \leq 0.0001)$ and from $75.9 \%$ in the morning and early afternoon to $70.6 \%$ from midnight to 0600 $(\mathrm{p} \leq 0.0019)$. Response accuracy was $8.6 \%$ higher for stimuli presented to the RVF than for stimuli presented to the LVF ( $\mathrm{p} \leq 0.0012$ ) and $22 \%$ higher for word stimuli than for nonword stimuli $(p \leq 0.003)$. Several interactions were also significant: number of days of sleep deprivation $\times$ word/nonword ( $\mathbf{p} \leq \mathbf{0 . 0 0 0 1 )}$; hour of day $x$ word/nonword ( $p \leq 0.0067)$; and number of days of sleep deprivation $\times$ visual hemifield $(p \leq 0.05$ ).

Response accuracy, averaged across the 6 subjects, is plotted in Figure 2 as a function of $72 \mathrm{~h}$ of sleep deprivation. Four curves are shown, representing data for each cell of the matrix word/nonword and RVF/LVF stimulation. Response accuracy decreased for all curves over $72 \mathrm{~h}$ of sleep deprivation, with the decrease marked by rhythmic variations. The slopes for nonword stimuli were significantly steeper than those for word stimuli ( $p \leq 0.001$ ). The slope of the curve for stimulation of the RVF by nonwords was steeper than that of the curve for stimulation of the LVF by nonwords ( $p \leq 0.05$ ). Although response accuracy at the beginning of the experiment was higher for stimulation of the RVF than of the LVF, by the latter part of the experiment, the curves clearly overlapped.

The four curves were analyzed by MCD. Regression analysis of the trend indicated that the analysis profile of response accuracy, unlike that of response lapses, was not uniform across the four curves. The overall trend ac- counted for $91 \%$ and $97 \%$ of the variance for nonwords, but only $48-59 \%$ of variance for words. The circadian component accounted for only $3.4-4.6 \%$ of the variance for nonwords, but $16 \%$ (LVF) and $35 \%$ (RVF) for words. The 2-cpd component accounted for only 1-2\% of the variance for nonwords, but $9 \%$ of variance for words.

Because the absolute decrease in accuracy of words was only 6-7\% over the 72-h period (Figure 2), the circadian component reflected only a $2 \%$ change in accuracy. The decrease in accuracy to nonwords was approximately $40 \%$, and the circadian component reflected approximately the same absolute change in accuracy $(2 \%)$; the overall trend reflected a change of approximately $35 \%$. A similar comment may be made when comparing word to nonword data in terms of the amount of variance accounted for by the 12-h or 2-cpd component.

The linear equations that best fit the trend accounted for approximately $88 \%$ of the variance of the decrease in accuracy. In contrast, the linear component accounted for only $28 \%$ (LVF) and $39 \%$ (RVF) of the variance of the words. The exponential equations accounted for approximately $85 \%$ of the nonword data and for $29 \%$ (LVF) and $37 \%$ (RVF) of the variance of the word data (Table 1B).

In summary, the decrease in accuracy for nonwords can be described best by a composite equation whose linear component is weighted approximately 20 times that of the rhythmic (mainly circadian) component, whereas the decrease in accuracy to words can be described best by

\section{RESPONSE ACCURACY AS A FUNCTION OF}

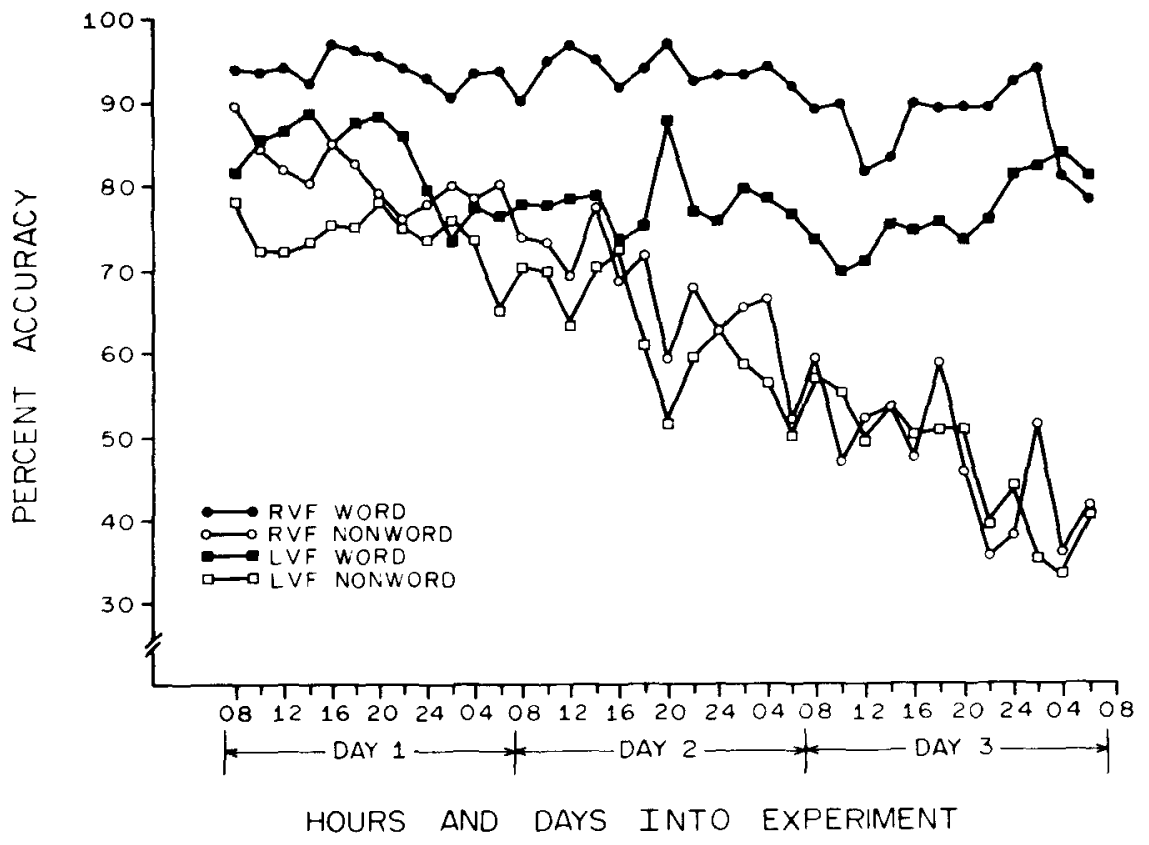

Figure 2. Response accuracy (in percent of correct responses), as a function of hours of progressive sleep deprivation, for the same four conditions shown in Figure 1. 
a composite equation whose monotonic (linear) and rhythmic components are weighted approximately equally. The linear component reflects a decrease in accuracy of approximately $33 \%$ for nonwords and of approximately 2-3\% for words, and the circadian component reflects a decrease of approximately $2-3 \%$ for both words and nonwords.

\section{Signal Detection Measures $\left(d^{\prime}\right.$ and $\beta$ )}

The appeal of the signal detection measures, $\mathrm{d}^{\prime}$ and $\beta$, is based on the assumption that they provide independent measures of the discriminability of a given pair of stimulus alternatives ( $\left.d^{\prime}\right)$, and the observer's decision criterion or bias toward one of the response alternatives $(\beta)$ (Green \& Swets, 1966).

Both $d^{\prime}$ and $\beta$ are based on the conditional probabilities of a correct discrimination (hit) and an incorrect discrimination (false alarm). Discriminability is represented by:

$$
\mathrm{d}^{\prime}=\mathbf{Z}\left(\mathbf{P}_{\text {false alarms }}\right)-\mathrm{Z}\left(\mathbf{P}_{\text {hits }}\right),
$$

where $Z\left(P_{\text {false alarms }}\right)$ and $Z\left(P_{\text {hits }}\right)$ are the normal deviates corresponding to $\mathbf{P}_{\text {false alarms }}$ and $P_{\text {hits }}$.

The decision criterion is represented by:

$$
\beta=\mathrm{f}\left(\mathbf{P}_{\text {hits }}\right) / \mathrm{f}\left(\mathbf{P}_{\text {false alarms }}\right),
$$

where $f\left(P_{\text {hits }}\right)$ and $f\left(P_{\text {false alarms }}\right)$ are the ordinates of the normal curve corresponding to $\boldsymbol{P}_{\text {hits }}$ and $\mathbf{P}_{\text {false alarms }}$.

$\mathrm{d}^{\prime}$ and $\beta$ were computed using correct responses to words as "hits" and incorrect responses to nonwords (responding "word" to a nonword) as "false alarms." d's and $\beta$ 's were computed separately for stimuli presented to the two hemifields and were analyzed by three-way ANOVAs: visual hemifields (RVF/LVF) $\times$ day $\times$ hour. All main effects are significant in affecting $d^{\prime}$, which decreases from Day 1 to Day 3 ( $p \leq 0.0001$ ) and over the hours of each day ( $p \leq 0.003$ ). In addition, $d^{\prime}$ is greater for responses to stimuli presented to the RVF than to the LVF ( $\mathrm{p} \leq 0.0001$ ). One significant interaction, day $X$ visual hemifield ( $p \leq 0.02)$, is described with the aid of Figure 3.

The only significant main effect affecting $\beta$ is visual hemifield. $\beta$ is greater for stimuli presented to the LVF than to the RVF ( $\mathrm{p} \leq 0.002$ ).

Figure 3 shows average $d^{\prime}$ values for 6 subjects plotted separately for the left and right visual fields. Both curves also show rhythmic variations. $d$ ' decreased from approximately 2.6 to 1.2 for RVF stimulation and from approximately 1.7 to 0.7 for LVF stimulation over 3 days of sleep deprivation (Figure 3 ). Linear equations were fitted separately to each of the two curves for each of the 6 subjects and accounted for approximately $52 \%$ of the variance for RVF stimulation and $57 \%$ of the variance for LVF stimulation. The slope for the RVF was steeper than that for the LVF ( $p \leq 0.05$ ).

Figure 4 shows average $\beta$ values for 6 subjects plotted separately for RVF and LVF. There is no systematic change in $\beta$ as a function of sleep deprivation for either curve ( $p \leq 0.425)$. There is, however, a difference between the two visual hemifields expressed in a higher $\beta$ for stimulation of the LVF than for stimulation of the RVF.

The $\mathrm{d}^{\prime}$ and $\beta$ curves were analyzed by MCD. Regres-

\section{D-PRIME AS A FUNCTION OF SLEEP DEPRIVATION}

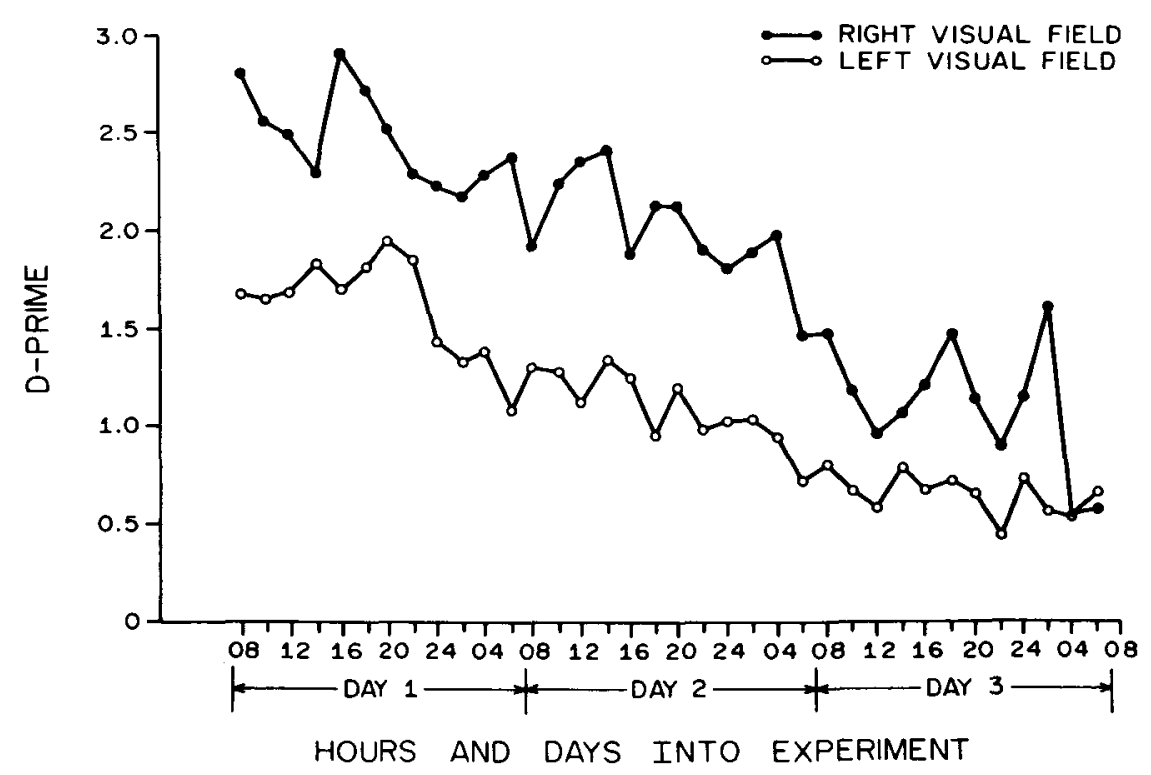

Figure 3. The signal detection measure of discriminability, $d^{\prime}$, as a function of hours of progressive sleep deprivation, for the two visual hemifields. 
BETA AS A FUNCTION OF

SLEEP DEPRIVATION

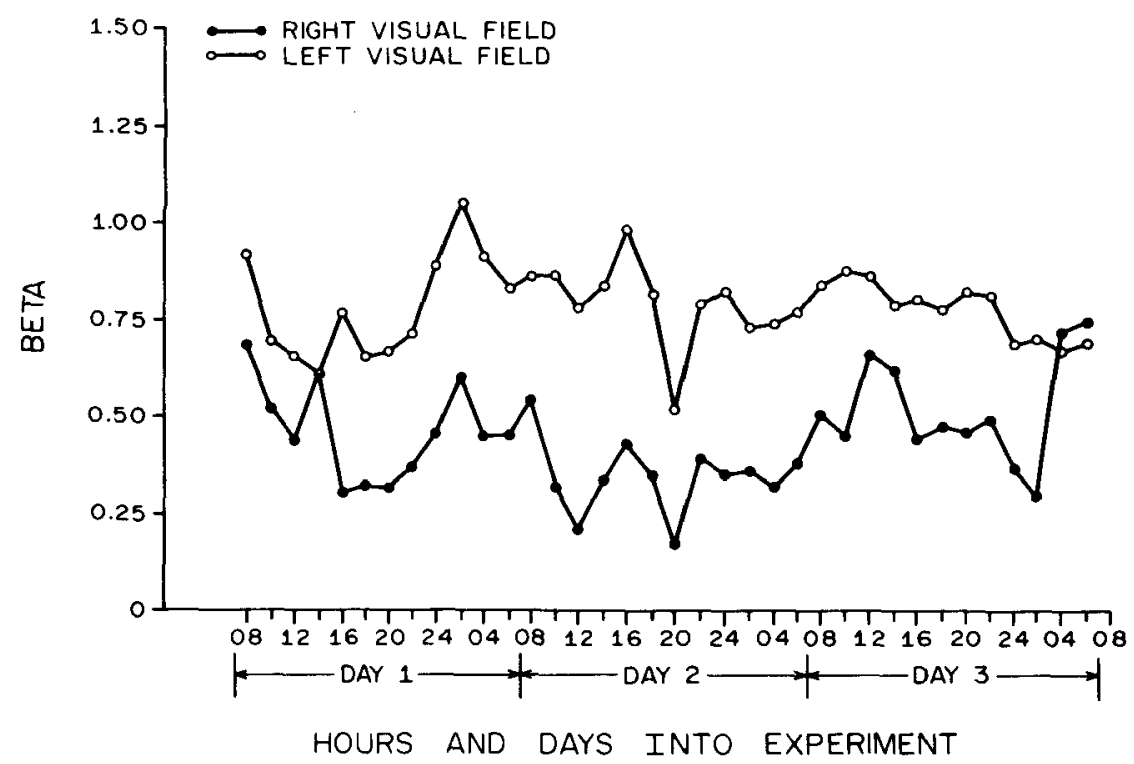

Figure 4. The signal detection measure of bias, $\beta$, as a function of hours of progressive sleep deprivation, for the two visual hemifields.

sion analysis indicated that the overall trend accounted for $86.1-92.3 \%$ (average of $89.2 \%$ ) of the variance for $\mathrm{d}^{\prime}$ (Table 1C), whereas the overall trend accounted for only $30 \%$ of the variance for $\beta$ (Table 1D). The slopes of the best-fitting equations do not differ significantly from zero $(\mathrm{F}=0.24, \mathrm{p} \leq 0.6 ; \mathrm{F}=2.9, \mathrm{p} \leq 0.10$, respectively) and account for almost none of the variance of $\beta$. The rhythmic components account for a large percentage of the $\beta$ variance and a much lower percentage of the $d^{\prime}$ variance.

In summary, the analysis of the $\mathrm{d}^{\prime}$ and the $\beta$ data indicate the following: (1) $d^{\prime}$ decreases as a function of sleep deprivation for stimuli presented to the RVF and LVF. The slope of the curve for RVF stimulation is significantly steeper than that for LVF stimulation. (2) Because of the initially lower $\mathrm{d}^{\prime}$ values associated with LVF stimulation and the general monotonic decrease over the 72-h period, discrimination by the LVF becomes essentially ineffective by the 44th-48th hour of sleep deprivation, as $\mathrm{d}^{\prime}$ decreases below 1.0. $\mathrm{d}^{\prime}$ is greater than 1.0 for stimulation of the RVF on the average, up to $65 \mathrm{~h}$ of sleep deprivation. (3) There is no systematic change in $\beta$ as a function of $72 \mathrm{~h}$ of sleep deprivation. (4) $\beta$ is higher for stimulation of the LVF than for the RVF. (5) There are strong rhythmic (circadian) variations in the $\beta$ data for stimulation of both of the visual hemifields.

\section{DISCUSSION}

The use of performance measures to test sleep deprivation is complicated by ambiguities in interpreting the meaning of observed deficits. Deficits may be due to microsleep, to decreased arousal/attention, and/or to increasing limitations on information processing capability. The nature of the lexical decision task in studying sleep-deprivation effects permits parsing the data into categories of response lapses (indications of arousal or attentional deficit) and accuracy measures (reflecting information processing deficit).

\section{Response Lapses}

Completeness of sleep loss in studies of sleep deprivation is often questionable. EEG analysis of subjects after prolonged wakefulness shows instances of brief "microsleeps" which are identified as Stage 1 sleep. If not immediately aroused, these microsleeps develop into sleep Stages 2 and 3. As sleep loss increases, the frequency of microsleeps also increases (Johnson \& Naitoh, 1974). The recognition of microsleeps led to the recording of response absences or omissions as a major dependent variable and behavioral symptom in studies of the effect of sleep loss on performance. It was argued that, as sleep deprivation continued, there would always be periods during which sleep-deprived subjects would perform accurately, alternating with periods of lapses. This approach has been labeled the "Walter Reed Lapse Hypothesis" (Williams, Lubin, \& Goodnow, 1959).

Kjellberg (1977a, 1977b, 1977c) questioned the adequacy of this hypothesis since it dealt with only one dimension of sleep-loss effects on performance (i.e., dearousal), whereas other decrements in performance also occur. Kjellberg (1977b) also argued that lapses were not dis- 
crete, abrupt periods of lowered arousal, but were usually characterized by gradual lowering of arousal, resulting in a response omission after reaching a critically low level.

If response lapses are indicative of microsleep or absence of arousal (dearousal), then they may represent either "nonreceipt" of the stimulus or "nonprocessing" of its information. The simplest expectation is that, during such periods, all stimuli would be "ignored" equally; hence, such a measure would be insensitive to stimulus variables requiring different levels or degrees of processing. The response lapse data reported here generally follow this prediction. There are small but significant differences in lapses to words versus nonwords and to stimuli presented to the RVF versus the LVF. This finding is consonant with a hypothesis which suggests reduced rather than total absence of arousal despite extreme fatigue and sleep loss.

Two of the four predictions of the Walter Reed Lapse Hypothesis are supported by the lapse data (Johnson \& Naitoh, 1974; Williams et al., 1959): (1) increase in the frequency of lapses with increasing sleep deprivation, and (2) presence of a circadian rhythm in the occurrences of response lapses. Frequency of lapses is higher in the period $0200-0800 \mathrm{~h}$ than at any other time of the day.

\section{Response Accuracy}

Buck and Gibbs (1972) suggested that changes in information-processing capability may be responsible for performance decrements in sleep deprivation. This suggestion is not necessarily incompatible with either the "dearousal" or "lowered arousal" explanations offered by the Walter Reed Lapse Hypothesis (1959) or by the Kjellberg (1977b) hypothesis. Subjects in progressive sleep deprivation may undergo changes in central nervous system functioning which occur in parallel, but at different rates. The effects of increasing instances of microsleep could result in general performance decrement and, specifically changes in the mechanisms involved with processing information.

Information-processing deficit may be reflected in differential effects of sleep loss on those stimulus variables that require different levels or types of information processing. If response accuracy, after removal of response lapses, represents the relative success of processing stimulus information, then it might be sensitive to those variables. Response accuracy does, in fact, show differential deficits for the four stimulus variables. These are: (1) A distinct superior response to stimuli presented to the RVF over stimuli presented to the LVF consistent throughout the $72 \mathrm{~h}$ of sleep deprivation. The difference in response accuracy favoring the RVF is supported in the literature, indicating functional left hemispheric superiority in a lexical decision task (Babkoff \& Ben-Uriah, 1983; Babkoff, Genser, \& Hegge, in press; Bradshaw, Hicks, \& Rose, 1979). (2) A decrease in discriminability of lexical stimuli over and above the response lapses. Errors in responding to words and nonwords increased over time. (3) The total decrease in response accuracy to nonwords was six to seven times greater than to words.
Although the decrease is composed of monotonic and rhythmic components, the relative weighting of these components differs with respect to words and nonwords. The major component accounting for the decrease in accuracy for nonwords is linear, whereas the monotonic and rhythmic components account equally for the decreased accuracy in words. (4) The rate of decrease in response accuracy was greater to stimuli preser. do the RVF than to the LVF.

The greater decrease in response accuracy to nonwords may indicate either increasing difficulty discriminating unfamiliar from highly familiar stimuli, or increasing bias toward responding with a familiar response (word) when discrimination becomes difficult and doubtful.

The greater rate of decrease in response accuracy to stimuli presented to the RVF than to the LVF is something of a dilemma. The usual prediction would be that the more difficult the discrimination, the more sensitive it is to stressors such as sleep deprivation. Since response accuracy for words and nonwords is generally higher for stimuli presented to the RVF than for stimuli presented to the LVF, it would indicate that the lexical decision is more difficult when stimuli are presented to the LVF. Hence, lexical decision to LVF stimulation should be more sensitive to sleep loss than should RVF stimulation. The opposite finding suggests that this argument may be too simplistic.

Among the multiple mechanisms underlying the response in a lexical decision task are sensory-perceptual and cognitive-linguistic pathways. External stimulus variables and response demands determine the relative weightings of the sensory-perceptual and the cognitive-linguistic contributions to each measured response. When the major limiting factor in determining the responses is sensoryperceptual, there is evidence of relative LVF superiority (Bradshaw et al., 1979; Sergent, 1982, 1983). When the major limiting factor is cognitive-linguistic, then RVF superiority is found (Bradshaw \& Gates, 1978; Bradshaw et al., 1979; Lieber, 1976; Sergent, 1982, 1983).

We suggest that during the course of sleep deprivation, the relative weighting of the factors determining response accuracy in a lexical decision task shifts from cognitivelinguistic to sensory-perceptual. When subjects are rested, the weighting for response determination is toward cognitive-linguistic, since stimuli are adequate for contrast and acuity. As sleep deprivation progresses, the sensory-perceptual factor begins to receive greater weighting. The stimulus parameters which were adequate for sensory-perceptual processing at the beginning of the experiment may not be after progressive sleep deprivation. Such a shift in weighting would be more detrimental to the processing of RVF stimuli than of LVF stimuli, resulting in the accelerated rate of decrease in accuracy for RVF toward the latter part of the experiment.

\section{Signal Detection Measures ( $d$ ' and $\beta$ )}

One of the consistent findings reported over the past 40 years is that performance decrements resulting from sleep loss are most severe when the tasks are prolonged, 
inherently boring, or lacking in incentives. Given the proper motivation, subjects can improve performance by "trying harder." In fact, the "end spurt" effect can be cited as evidence for the belief that conscious effort can improve performance considerably for short time periods (Thorne et al., 1983).

The interpretation of these phenomena is unclear. It can be hypothesized that sleep loss leaves the basic information-processing mechanisms intact and capable of functioning for short periods but only with increased effort. If the effort required for performance maintenance of long tasks must be continued over longer periods, deficit becomes evident (Johnson \& Naitoh, 1974; Meddis, 1982). Alternatively, it can be hypothesized that sleep deprivation affects the information-processing mechanisms adversely. The lack of consistent findings relating performance deficits to proposed mechanisms may reflect the inability of previous and present instruments to differentiate between these two possibilities.

Signal detection theory may be applicable for data interpretation if it can differentiate discriminability changes $\left(d^{\prime}\right)$ from motivational changes $(\beta)$. Horne, Anderson, and Wilkinson (1983) report the effects of $60 \mathrm{~h}$ of sleep deprivation on signal detection measures using an auditory vigilance task administered five times every $24 \mathrm{~h}$. Their data show $\mathrm{d}^{\prime}$ as a stepwise declining function, dropping mainly during the night and leveling off during the daytime. The decrease in $\mathrm{d}^{\prime}$ from 4.25 to approximately 2.5 over their 60 -h period was due to a decrease in hit rate from approximately $92 \%$ to less than $60 \%$, whereas change in false alarm rate is minimal (around $1-2 \%$ ). The $\beta$ index shows an erratic but increasing trend. These results are interpreted to mean that sleep deprivation mainly affects $\mathrm{d}^{\prime}$, the intrinsic capacity to discriminate signals from noise, and does not affect $\beta$, the change in willingness to respond.

After studying the effects of sleep loss on the performance of a modified vigilance task, Deaton, Tobias, and Wilkinson (1971) concluded that $\mathbf{d}^{\prime}$ and $\beta$ were effective differentiators. Naitoh (1983), however, criticized the application of signal detection theory to measures of vigilance performance in sleep-deprived subjects on the basis that, for signal detection to be meaningful, the subject must observe and attend to all signals over the entire test session. Jenson, Pickett, and Stenson (1965) had argued that reduced responding caused by fatigue and/or boredom can produce spurious $\mathrm{d}^{\prime}$ and $\beta$ values which can be misinterpreted as changes in sensitivity and criterion.

Nonattention to signals can result in decreased hits which, if false alarm rate is low and changes little (as is usually the case in a vigilance task), would reduce the calculated $\mathrm{d}^{\prime}$, inappropriately implying reduced sensitivity. Decreased attention to signals, as a consequence of microsleeps, would result in reduced hits, raising questions regarding the meaning of a decreased $\mathrm{d}^{\prime}$ and an increasing or unaffected $\beta$ as sleep deprivation progresses.
The design of a vigilance experiment usually involves the detection of and response to a very low probability signal in the presence of high probability nonsignal stimuli within fairly long sessions. It is not easy to differentiate a miss caused by decreasing discriminability of signal and nonsignal from a miss caused by increased inattention or microsleep. In fact, the main source of the decline in $\mathrm{d}^{\prime}$ as sleep deprivation progresses is the decrease in hits rather than the increase in false alarms (see, e.g., Horne et al., 1983). A response lapse and a "missed" signal are undifferentiable with the vigilance paradigm used by Wilkinson and his colleagues (Naitoh, 1983).

The design of the experiment reported here required the subject to respond to the two types of stimuli, words and nonwords, by different responses. Stimuli to which no response occurred reflect the periods of reduced attention or microsleep and were removed from calculation of $d^{\prime}$ and $\beta$. Naitoh's (1983) criticism of the use of signal detection measures in sleep deprivation experiments is not applicable to the present design.

The decline in $\mathrm{d}^{\prime}$ stems from a small decrease in hit rate and a very large increase in false alarms as sleep deprivation accumulates. The decrease in $d^{\prime}$ is found for stimulation in both visual hemifields and reaches a level of less than 1 (very reduced discrimination) for the LVF by the 44th to the 48th $\mathrm{h}$ of sleep deprivation. Although the $d^{\prime}$ for RVF stimulation remains greater than 1 for an additional $20 \mathrm{~h}$, the slope of the function relating reduction of $d^{\prime}$ to sleep deprivation for RVF stimulation is steeper than for LVF.

$\beta$ was less than 1 for both visual fields over the entire experimental period. This value of $\beta$ is interpreted as a liberal criterion and, in the context of our experiment, represents a bias to respond "word" (signal) rather than "nonword" (noise) when in doubt as to stimulus identity. The significant main effect in the $\beta$ data was visual field, with LVF stimuli consistently showing higher values of $\beta$ than RVF stimuli. This implies a greater tendency to respond "word" when in doubt, if the stimulus is presented to the RVF.

The insensitivity of $\beta$ to progressive sleep loss and its difference between the two visual fields implies a basic lexical response bias toward more frequent word processing by the left hemisphere than by the right. The circadian variation of $\beta$ indicates a high correlation of this measure with the basic rest/activity cycle. Since performance measures are known to be correlated with circadian rhythmicity (Angus \& Heslegrave, 1985; Monk et al., 1985; Thorne et al., 1983), the large monotonic decrease in performance, as reflected by the decrease in $\mathrm{d}^{\prime}$, indicates a mechanism becoming increasingly fatigued with time.

These results address the issue of changes in discriminability versus changes in response bias. The large monotonic changes in $\mathrm{d}^{\prime}$ accompanied by the relative (monotonic) stability in $\beta$ indicate that the major effect of sleep loss in a lexical task is to reduce discriminability. 


\section{REFERENCES}

Angus, R. G., \& Heslegrave, R. J. (1985). Effects of sleep loss on sustained cognitive performance during a command and control simulation. Behavior Research Methods, Instruments, \& Computers, 17, 55-67.

BABKOFF, H., \& BEN-URIAH, Y. (1983). Lexical decision time as a function of visual field and stimulus probability. Cortex, 19, 13-30.

Babkoff, H., Genser, S. G., \& Hegge, F. W. (in press). Lexical decision, parafoveal eccentricity and visual hemifield. Cortex.

Babkoff, H., Genser, S. G., Sing, H. C., Hegge, F. W., \& Thorne, D. R. (1985). The effects of sleep loss and work load on selected psychological and physiological variables: III. Perceptual distortions and hallucinations. Washington, DC: Walter Reed Army Institute of Research, Department of Behavioral Biology. (Technical Report in Preparation)

Babkoff, H., Thorne, D. R., Sing, H. C., Genser, S. G., Taube, S. L., \& HEGGE, F. W. (1985). Dynamic changes in work/rest duty cycles in a study of sleep deprivation. Behavior Research Methods, Instruments, \& Computers, 17, 604-613.

BELENKY, G. (1979). Unusual visual experiences reported by subjects in the British Army study of sustained operations, Exercise Early Call. Military Medicine, 144, 695-696.

BRADShaw, G. J., \& GaTES, A. (1978). Visual field differences in verbal tasks. Brain \& Language, 4, 166-187.

Bradshaw, G. J., Hicks, R. E., \& Rose, B. (1979). Lexical discrimination and letter-string identification in the two visual fields. Brain \& Language, 25, 353-370.

Buck, L., \& GiBBs, C. B. (1972). Sleep loss and information processing. In W. P. Colquhoun (Ed.), Aspects of human efficiency. London: English Universities Press.

Deaton, M., Tobias, J. S., \& Wilkinson, R. T. (1971). The effects of sleep deprivation signal detection parameters. Quarterly Journal of Experimental Psychology, 23, 449-452.

Green, D. M., \& SwETS, J. A. (1966). Signal detection theory and psychophysics. New York: Wiley.

HEISE, D. R. (1978). Computer assisted analysis of social action: Use of program interact and survey UNC Technical Report No. 75. Chapel Hill, NC: Institute for Research in Social Science, University of North Carolina.

Herscovitch, J., \& Broughton, R. (1981). Performance deficits following short term partial sleep deprivation and subsequent recovery oversleeping. Canadian Journal of Psychology, 35, 309-322.

HoRNE, J. A. (1983). Human sleep and tissue restitution: Some qualifications and doubts. Clinical Science, 65, 569-578.

Horne, J. A., ANDERson, N. R., \& Wilkinson, R. T. (1983). Effects of sleep deprivation on signal detection measures of vigilance: Implications for sleep function. Sleep, 6, 347-358.

Jenson, H. J., Pickett, R. M., \& Stenson, H. H. (1965). The clinical observing rate and decision process in vigilance. Human Factors, 7, 107-128.

JoHNSON, L. C. (1982). Sleep deprivation and performance. In W. B. Webb (Ed.), Sleep, biological rhythms and performance. New York: Wiley.

Johnson, L. C., \& NaIToH, P. (1974). The operational consequences of sleep deprivation and sleep deficit AGARDograph AF-193. NATO Advisory Group for Aerospace Research and Development. London, England: Technical Editing and Reproduction.

KJELLBERG, A. (1977a). Sleep deprivation and some aspects of performance: I. Problems of arousal changes. Waking \& Sleeping, 1 , 139-143.

KJELLBERG, A. (1977b). Sleep deprivation and some aspects of performance: II. Lapses and other attentional effects. Waking \& Sleeping. 1, 145-148.
KJellberg, A., (1977c). Sleep deprivation and some aspects of performance: III. Motivation, comment and conclusion. Waking \& Sleeping, 1, 149-153.

Lieber, L. (1976). Lexical decisions in the right and left cerebral hemispheres. Brain \& Language, 3, 443-450.

MEDDIs, R. (1982). Cognitive dysfunction following loss of sleep. In E. Burton (Ed.), The Pathology and Psychology of Cognition. London: Methuen

Monk. T. H., Fookson, J. E., Kream, J., Moline, M. L., Pollak, C. P., \& Weitzman, M. B. (1985). Circadian factors during sustained performance: Background and methodology. Behavior Research Methods, Instruments, \& Computers, 17, 19-26.

NaItoH, P. (1983). Signal detection theory as applied to vigilance performance of sleep-deprived subjects. Sleep, 6, 359-361.

Patrick, G. T. W., \& Gilbert, J. A. (1896). On the effects of loss of sleep. Psychological Review, 5, 476-483.

Redmond, D. P., Sing, H. C., \& Hegge, F. W. (1982). Biological time series analysis using complex demodulation. In F. W. Brown \& R. C. Graeber (Eds.), Rhythmic aspects of behavior. Hillsdale, NJ: Erlbaum.

SERGENT, J. (1982). Theoretical and methodological consequences of variations in exposure duration in visual laterality studies. Perception \& Psychophysics, 31, 451-461.

SERGENT, J. (1983). The effects of sensory limitations of hemispheric processing. Canadian Journal of Psychology, 37, 345-366.

Sing, H. C., Genser, S. G., BabKofF, H., Thorne, D. R., \& HegGe, F. W. (1984). Complex demodulation - a technique for assessing periodic components in sequentially sampled data. Proceedings of the 29 th Conference on Design of Experiments in Army Research Development and Testing (Army Research Office Technical Report No. 84-2). Bethesda, MD: Uniformed Services University of the Health Sciences.

Sing, H. C., Redmond, D. P., \& Hegge, F. W. (1980). Multiple complex demodulation: A method for rhythmic analysis of physiological and biological data. Proceedings of the 4th Annual Symposium of Computer Application in Medical Care (pp. 151-158). New York: Institute of Electrical \& Electronic Engineers.

Sing, H. C., Thorne, D. R., Hegge, F. W., \& Babkoff, H. (1985). Trend and rhythm analysis of time-series data using complex demodulation. Behavior Research Methods, Instruments, \& Computers, 17, 623-629.

Thorne, D. R., Genser, S. G., Sing, H. C., \& HegGe, F. W. (1983). Plumbing human performance limits during 72 hours of high task load. In S. E. Forshaw (Ed.), Proceedings of the 24th Defense Group Seminar on the Human as a Limiting Element in Military Systems: Volume $I$ (NATO-DRG Report No. DS-A-DR(83) 170, pp. 17-40). Toronto: NATO Defence Research Group.

WILKINSON, R. T. (1964). Effect of up to 60 hours of sleep deprivation on different types of work. Ergonomics, 7, 175-186.

Williams, H. L., LUBin, A., \& GoodNow, J. J. (1959). Impaired performance with acute sleep loss. Psychological Monograph, 73(No. 484).

\section{NOTE}

1. The criterion defining a response lapse as $3 \mathrm{sec}$ of nonresponding is more conservative than the criterion used by Herscovitch and Broughton (1981), who defined a "gap" (lapse) as a 1-sec lack of responding. Their paradigm (a four-choice serial reaction-time test) differed from the present task also in that a stimulus was presented approximately $120 \mathrm{msec}$ after the previous response, so that $1 \mathrm{sec}$ of nonresponding reflected eight to nine missed stimuli. In the present paradigm, a response lapse reflected $3 \mathrm{sec}$ of nonresponding to a single stimulus. 